\title{
A COMPARATIVE MODEL STUDY BETWEEN THE SURVIVABILITY OF DENTAL IMPLANTS VERSUS DENTAL AUTOTRANSPLANTS, LEADING TO A SYSTEMATIC REVIEW
}

KEY WORDS:

\begin{tabular}{ll}
\hline Alcobia IM & Instituto Universitário Egas Moniz-2829-511 Monte da Caparica, Portugal. \\
\hline Gomes J & $\begin{array}{l}\text { Centro de Investigação Interdisciplinar Egas Moniz - 2829-511 Monte da } \\
\text { Caparica, Portugal., Universitat Internacional de Catalunya - 08195 Sant } \\
\text { Cugat delVallès,Barcelona,España. }\end{array}$ \\
\hline Maia P & $\begin{array}{l}\text { Centro de Investigação Interdisciplinar Egas Moniz - 2829-511 Monte da } \\
\text { Caparica,Portugal. }\end{array}$ \\
\hline Proença L & $\begin{array}{l}\text { Centro de Investigação Interdisciplinar Egas Moniz - 2829-511 Monte da } \\
\text { Caparica,Portugal. }\end{array}$ \\
\hline Marques JF & Faculdade de Farmácia da Universidade de Lisboa- 1649-003 Lisboa. \\
\hline Marques J* & $\begin{array}{l}\text { Centro de Investigação Interdisciplinar Egas Moniz - 2829-511 Monte da } \\
\text { Caparica, Portugal., Hospital da Luz - 2900-722 Setúbal, Portugal. } \\
\text { *Corresponding Author }\end{array}$ \\
\hline \hline
\end{tabular}

E Aim: The aim of this Systematic Review is to compare the survival rate of teeth autotransplantation with immediate implant placement in cases of singular loss of teeth and obtain a clinical recommendation for similar cases.

Materials And Methods: The research will be conducted by two independent reviewers in PubMed search and it will include studies from January 2017 to December 2020. The survival rate of both immediate implant placement and tooth autotransplantation will be evaluated in different follow-up times and the information compiled in several metaanalyses.

\section{INTRODUCTION}

Tooth auto trasplantation can be defined as the transplantation of a tooth from one site to another in the same individual (Machado et al., 2016). Reports of tooth transplantation are dated from many centuries ago but only in the $20^{\text {th }}$ century were autotransplants considered and executed with relative success (Armstrong et al., 2020). Several reviews state the ideal conditions and protocols to perform this procedure. The protocol can vary in several aspects, such as: the root formation level of the donor tooth; the splinting material and timeline; the use or not of a surgical guide and $\mathrm{a} 3 \mathrm{D}$ model of the donor tooth which allows for a lower extra alveolar time of that donor tooth, improving the prognosis (Yu et al., 2017).

With the same purpose in mind, to rehabilitate a lost tooth, there were also developed dental implants, structures inserted in the upper or lower jaw bone to which a crown will be attached (Howe et al., 2019). The concept of immediate implant placement refers to an implant placed right after the extraction of the tooth it is ought to replace (Antetomaso \& Kumar 2018).

On that account, immediate implant placement and tooth autotransplantation are two techniques developed for similar clinical scenarios and therefore, the need for a clinical recommendation between the techniques is evident.

\section{MATERIALS AND METHODS \\ Protocol}

This Systematic Review will report according to the PRISMA statement and answers the following PICO question: In cases of singular loss of teeth in the posterior mandibular region (population), which is the rehabilitation option with the best prognosis (outcome), tooth autotransplantation or immediate implant placement (intervention and comparison)?

The research will be conducted by two reviewers, independently, in the database PubMed search using relevant keywords and include studies from January 2017 to December 2020.

|www.worldwidejournals.com

\section{Aim}

The aim of this study is to compare teeth autotransplantation with immediate implant placement in cases of singular loss of teeth and obtain a clinical recommendation for similar cases.

\section{Inclusion And Exclusion Criteria:}

The inclusion criteria are the following: studies in English; studies with minimum l-year follow-up; studies which do not include patients with systemic diseases; no limitations were considered regarding the donor tooth for the transplant intervention.

The exclusion criteria are as follows: studies which do not include the location of the intervention or the sample includes only interventions outside the posterior mandibular region; studies that include patients with systemic diseases or undergoing medicine that influences bone metabolism (e.g., biphosphates, radiotherapy); animal studies; studies with samples related to multiple rehabilitation instead of single site rehabilitation.

Quality Assessment OfThe Studies Included

In order to evaluate the risk of bias, the AXIS guideline will be used. The information then compiled with the use of "RobVis" tool and described as low, moderate or high risk of bias.

\section{Data Extraction}

The data included will follow an independent research by the reviewers and a discussion to settle any conflicts.

After a title analysis, an assessment of the abstracts will be conducted. All studies that are not excluded based on the abstract assessment will proceed to a full test assessment. The studies that fulfill the inclusion criteria and are not eliminated by the exclusion criteria will be included in this Systematic Review.

The data extracted will be devised in two independent analyses, one for immediate implant placement and one for teeth autotransplantation. At a follow-up time of 6 months, 1 year, 5 years and 10 years, the survival rates of the 
interventions will be recorded and all information then compiled in several meta-analysis.

In order to compare the samples, a "Z" test will be conducted. If there is not a statistical significant difference between the samples $(p>0,05)$, a comparison will be made and conclusions extracted.

All meta-analysis performed will follow a binominal randomized model with maximum restricted likelihood using the program "Open meta-analyst" with a confidence level of $95 \%$.

A clinical recommendation will then be carried out based on the findings of this Systematic Review.

\section{REFERENCES}

1. Antetomaso,J., \& Kumar, S. (2018). Survival Rate of Delayed Implants Placed in Healed Extraction Sockets is Significantly Higher Than That of Immediate Implants Placed in Fresh Extraction Sockets. Journal of Evidence Based Dental Practice, 18(1),76-78.https://doi.org/10.1016/j.jebdp.2017.12.003

2. Armstrong, L., O'Reilly, C., \& Ahmed, B. (2020). Autotransplantation of third molars: a literature review and preliminary protocols. British Dental Journal, 228(4),247-251.https://doi.org/10.1038/s41415-020-1264-9

3. Howe, M. S., Keys, W., \& Richards, D. (2019). Long-term (10-year) dental implant survival: A systematic review and sensitivity meta-analysis. Journal of Dentistry, 84,9-21.https://doi.org/10.1016/j.jdent.2019.03.008

4. Machado, L., do Nascimento, R., Ferreira, D., Mattos, C., \& Vilella, O. (2016) Long-term prognosis of tooth autotransplantation: a systematic review and meta-analysis. International Journal of Oral and Maxillofacial Surgery, 45(5), meta-analysis. International Journal of Oral and Maxil/o/

5. Yu, H., Jia, P., Lv, Z., \& Qiu, L. (2017). Autotransplantation of third molars with completely formed roots into surgically created sockets and fresh extraction sockets: a 10-year comparative study. International Journal of Oral and Maxillofacial Surgery, 46(4), 531-538. https://doi.org/10.1016/ j.ijom.2016.12.007 